\title{
The changing relationship between neurology and general practice in the UK
}

\author{
Paul Morrish
}

\begin{abstract}
The full involvement of primary care in an organised and balanced health service is vital to neurological patients' health. However, the available evidence suggests that GPs are, if anything, withdrawing or being pushed out of caring for this patient group. This article tries to identify why this may be happening, considers the implications, and suggests alternative ways forward.
\end{abstract}

\begin{abstract}
'The survey reveals that $35 \%$ of our members who were diagnosed more than 10 years ago were diagnosed by their GP. Things are getting better, with more people with Parkinson's being diagnosed by a specialist but, still, one in five people diagnosed in the last year were told they have Parkinson's by their GP.' (From Parkinson's News) ${ }^{1}$
\end{abstract}

\section{INTRODUCTION}

It is in the interest of patients for primary care to take a central role in the diagnosis and, when necessary, the long-term management of neurological and neurodegenerative disease. As the population ages, the prevalence of neurodegenerative disease is increasing, and so it will take up a much greater proportion of NHS time and expense. From the author's perspective as an NHS neurologist, it appears that general practice in the UK is being ushered out and may also be opting out of the diagnosis and management of neurological symptoms and disease. This is detrimental to the care of neurological patients, and this provocative statement will be justified with the aim not to offend but to highlight a problem and look for solutions.

\section{WHY SHOULD GPS TAKE AN INTEREST IN NEUROLOGY?}

Neurological presentation to general practice is common and makes up around $7 \%$ of consultations. One study reports that $0.6 \%$ of the population will have had neurological disease diagnosed in the previous year, while $6 \%$ will have had a neurological condition at some time. ${ }^{2}$ These figures may be underestimates; the study by MacDonald et $a{ }^{2}$ included stroke but excluded Bell's palsy, benign positional vertigo, back pain, migraine, dementia, and tension headache (all of which are common presentations to GPs and referrals to neurology). Neurological illnesses are frequently disabling and only rarely do they go away. They have an impact on the individual's role in the community and on all those around them. Neurological illnesses can result in dependency, impaired quality of life, and premature death. Who better to manage and coordinate their 
long-term care than the GP? A UK GP recently wrote (in a neurology journal) that:

'Family physicians are ideally placed to identify patients with epilepsy whose management needs optimising. This is because community-held disease registers can identify everyone with epilepsy and then be used to proactively offer a review of their care. In particular vulnerable groups such as the elderly, people with learning disability, those with psychiatric illness, and highrisk patients with problems of drug or alcohol addiction may be reached. Opportunity may also be taken in primary care to address social needs such as relationship breakdown, unemployment, stigma, isolation, poor self-esteem, and so on. These are issues which may be forgotten in a busy neurology clinic.' 3

This campaigning statement for the role of the GP in the long-term care of epilepsy could also apply to Parkinson's disease, multiple sclerosis (MS), stroke, and dementia.

In addition to longer-term management, there is also the question of where newly presenting neurological symptoms are best investigated, diagnosed, and managed. Neurologists are experts in neurological disease in its multiple and rare presentations and yet, perhaps paradoxically, they may not be the best people to consider whether to investigate common symptoms. Close acquaintance with serious neurological illness could be a distracting background for the assessment of modest and everyday neurological symptoms like headache, tiredness, and myalgia.

The GP, with long-term knowledge of the patient, their family, and their social circumstances, might opt to watch and wait, but the neurologist, who will usually only have hospital notes and the patient in front of them, might investigate. The neurologist may only have (or worse, may only be interested in) the bare medical essentials, and may not see beyond the purest medical explanation of the patient's symptoms (in fairness to them, they may not be trained to see these). Secondary and tertiary care investigation of medically unexplained symptoms may not be in the patient's interest, ${ }^{4}$ potentially reinforcing the idea that 'something must be wrong'. A referral to neurology, intended to reassure the patient and doctor that the symptoms are benign, could result in multiple investigations and a worrying false positive or two.

\section{WHAT IS THE EVIDENCE THAT GENERAL PRACTICE IS BEING EXCLUDED OR IS OPTING OUT?}

Neurology in the UK has been, and still is, astonishingly understaffed in comparison to US and other European populations, which is one of the arguments cited by the Association of British Neurologists for its expansion. ${ }^{5}$ The average population per neurologist in the US is 22000 , in France it is 38000 , and in the UK in 2005 (and after considerable expansion) it was 117000 . Stevens wrote of the workload of an English neurologist in 1989 when there was one neurologist in the UK per $277000 .{ }^{6}$ His waiting list was 22 weeks and after dedicated observation over several years he commented (and one can sense his besieged position from the language) that 'a shorter waiting list unleashed a flood of additional referrals'. Between 1993 and 2005 the number of consultant neurologists in England, Wales, and Northern Ireland increased from 224 to $465,{ }^{7}$ and, in line with government targets, waiting lists have come down so that few neurology units breach the current 9-week rule.

The author's experience in consultant posts in Sussex and Gloucestershire has supported Stevens' comments. In Brighton, as the waiting list decreased between 1997 and 2001, there was a rise in new patient referrals from 800 to 1800 per year. In Gloucestershire it has risen from just over 1000 in 1989 to over 4000 in 2006, and over the past year by a further $10 \%$. This experience prompted the author to look at the national picture of referrals to neurology. ${ }^{8}$ In the 2 years between 2003 and 2005, there was a $24 \%$ increase in new outpatient attendances in neurology in England, from 5.8 new patient appointments per 1000 population (aged $>15$ years) to 7.2. The rise appears to have taken place throughout all primary care trusts (PCTs), regardless of the pre-existing number of referrals per head of population. In the 31 PCTs of Greater London, where in 2003-2004 the average number was already comparatively high at 8.1 per 1000 , new patient referrals increased by $22 \%$ to 9.9 per 1000 in 2005-2006. It is important to add that it is not only primary care that appears to be referring more. There has been a similar rise in referrals to neurology from within secondary care. ${ }^{8}$

In London, despite the relatively high number in 2003-2004, referral numbers carried on rising. The implication is that neurology referrals seem likely to go on increasing throughout the country, to London levels

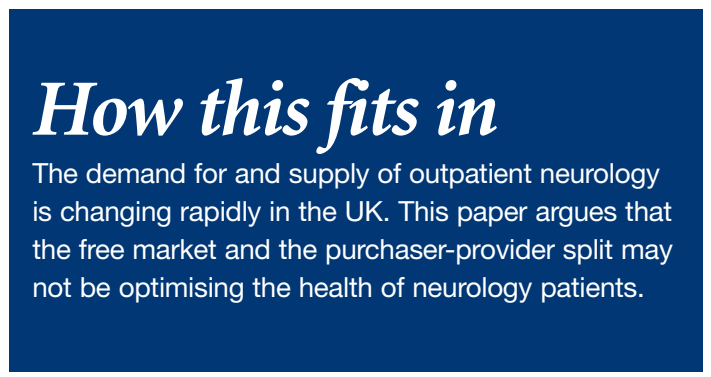


or higher. The other observation in that study ${ }^{8}$ was the striking regional disparity (by PCT of residence) in new patients seen in neurology, and in some PCTs six times as many patients per head of population were seen by neurology as in others. This matched the regional disparity in distribution of neurologists, with one neurologist per 60000 or fewer in parts of London, while elsewhere in the UK there may be only one consultant for a population of over $300000 .^{7}$ In general, the populations living close to neurology centres (for example, London, Cambridge, Newcastle, and Sheffield) were most likely to be seen in neurology outpatients. It seems unlikely that there is six times as much neurological illness close to the neurology centres, and one can speculate that the healthy supply (or could it be an unhealthy over-supply?) of neurologists in these areas is facilitating referral of many more patients to neurology.

\section{WHY IS THIS HAPPENING?}

This change is unlikely to be explained by a corresponding massive change in incidence of disease or of symptom presentation in primary care. So while the GP or other specialist may be referring only one in every 100 patients with neurological symptoms, there are unarguably very many more new patient referrals to neurology than there were a few years ago. It may be that patients once referred to other specialties (for example, epilepsy, transient ischaemic attack, stroke, dementia, and learning disability) are now referred to neurology, and perhaps general practice always did refer these patients somewhere; for example, to general medicine, rheumatology, or care of older people. Large practices with a long record of their referrals might provide the answer. A hospital policy of discharging and relabelling the follow-ups as new patients when re-referred might also confound the picture, although nationally the ratio of new to followup appointments has not been changing. ${ }^{8}$

Primary care is a huge specialty and neurology a small one, so a very small change in referral practice in primary care makes a huge difference to the numbers received in neurology. For example, if $4 \%$ instead of $3 \%$ of headaches seen in primary care (where the annual consultation rate for headache is 4.4 per 100 patients, ${ }^{9}$ and the population 60 million) were to be referred to neurology, there would be 105600 headache referrals per year instead of 79200 ; that increase would be likely to need around 30 more neurologists (because each sees around 1000 new patients per year in the NHS). Headache made up $15 \%$ of new patient referrals in Stevens' study, ${ }^{6}$ and if this apparent small increase in referral (from $3 \%$ to $4 \%)$ was applied to all neurological consultations, the number of neurologists needed would rise from 528 to 704. This may well explain why neurologists can feel 'a flood of additional referrals' yet GPs don't perceive any significant change in their practice. The seemingly relentless rise felt by neurology might be interpreted as primary care being a less effective gatekeeper to neurology than it once was. Equally, however, it could be viewed as the appropriate response to increasing pressure to refer to secondary care, a better targeted referral to an appropriate specialty, and a confirmation of how stunningly effective a gatekeeper for neurology primary care actually has been.

Although the picture is complex, it is important to look for explanations if we are to provide good and equitable neurological care. One obvious explanation is that by removing the previous barrier of a very long waiting list (in some areas the waiting list was over 2 years in the 1980s), it is easier to refer, more patients are being referred, and neurological provision in outpatients has, through a government waiting list directive, reacted to that demand. The more neurologists in post, the more clinic spaces available, the lower the barrier to referral, and the more patients are referred.

But other things have changed in society and in the organisation of the health service since 1989, and there are other explanations. Patients are informed and misinformed by the internet and the media, and are often keen to request a second opinion. They know that modern scanning techniques can detect serious illness and that they cannot have a scan without a referral. An audit among Brighton GPs showed that $25 \%$ of neurology referrals were felt to be primarily instigated by the patient. ${ }^{10} \mathrm{~A}$ system that was designed and once provided according to medical need seems now to be driven, at least in part, by patient demand.

In 1989 Stevens classed 5\% of patients referred to his neurology clinic as having 'disorders of function', problems where there is no clear-cut detectable cause or where an alternative label would be inappropriate. A further $9 \%$ were labelled as 'sensory symptoms ?cause, non-organic symptoms, post-viral symptoms and depression'. In 1975, what he then categorised as 'medically unexplained symptoms (MUS), migraine, vertigo and syncope' made up $25 \%$ of his new outpatients and in 1997 it was 39\% (Stevens, personal communication, 2005). In 2000 in Scotland, 30\% of patients referred to neurology were categorised as MUS. ${ }^{11}$ The comparison between Gloucester in 1975 and 1997 and Edinburgh in 2000 does not necessarily mean that twice (or perhaps even six times) as many patients without neurological disease are being referred to neurology clinics; there are too many confounding issues to be able to make such a statement (for example, Stevens being more likely to label symptoms as neurological than clinicians in Edinburgh), but it is suspicious. It also wouldn't be 
surprising when the rise in referrals to neurology is compared to the small change in prevalence of neurological disease over the past 20 years. It may be of note that neurologists are now showing an academic interest in patients with MUS, ${ }^{11}$ and it begs the questions of whether neurology is the right place to refer them or whether they should be referred at all.

GPs (and junior and senior hospital doctors) can lack confidence in neurology. ${ }^{12}$ This 'neurophobia' begins in medical school, ${ }^{13}$ and the combination of neurophobia and litigation phobia may make it (and it may actually be) safer to refer than to watch and wait. GPs with only one or two chronic neurology patients on their list may well feel that their experience is not enough to manage those patients well, and they may prefer to rely on secondary care.

The National Institute for Health and Clinical Excellence (NICE) has set a series of guidelines in neurological conditions. For Parkinson's disease the guidance is that if your doctor thinks you may have Parkinson's disease 'you should see the specialist within 6 weeks'. ${ }^{14}$ For suspected MS the guidance is that 'the patient should be referred to a specialist and seen rapidly', ${ }^{15}$ and in cases of suspected epilepsy the guidance is for patients to be seen urgently by a specialist. ${ }^{16}$ Many symptoms can be interpreted (and indeed could present) as suspected Parkinson's disease (for example, frozen shoulder, or fatigue), suspected MS (tingling legs), or suspected epilepsy (fainting), and the guidance encourages referral and leaves open to complaint any GP who opts to watch judiciously and wait or to have the temerity to make the diagnosis themselves. A recent review omits a role for primary care in the long-term management of patients with neurological disability. ${ }^{17}$ NICE also advises regular follow-up by a specialist in each condition. These articles of guidance do not come out of nothing; each committee justifies its position by pointing out the potential for delay in neurological diagnosis, the potential for misdiagnosis (although a recent paper shows that specialists in movement disorders also misdiagnose parkinsonian syndromes at an early stage), ${ }^{18}$ and the high level of expertise needed to manage the conditions. The committees are made up of patient representatives and a group of professionals biased towards those with an interest in the condition. Each NICE review includes primary care members, but the importance in making a diagnosis in primary care, and the longer-term benefits (for patient and GP) in GPs taking the diagnostic and continuing care role seem absent. There seems little recognition of potential problems (for the individuals as well as for the healthcare system) in referring all 'suspected' Parkinson's disease, MS, and epilepsy to secondary care, and for secondary care to follow up all the diagnosed patients at regular intervals.
Some responsibility for the growth in referrals lies with neurologists too. Neurologists may be the keyholders to computed tomography (CT) and magnetic resonance imaging (MRI) and the GP can not have one without the other. GPs confronted on a daily basis with the 'I must have a scan for my headache' health belief can have little option but to refer. Neurologists may make the assumption that the GP is not interested in or not able to manage the long-term care and that secondary care expertise is inevitably better. There is also a potential conflict for those neurologists working both in the NHS and in private practice; building a private practice is unlikely to be harmed by encouraging referral for minor neurological symptoms and dependence on a specialist for continuing care. Medicalisation and over-specialisation of common symptoms (such as migraine and restless legs) does not help either, although blame may be more widely spread between patient organisations, specialists, the media, and the pharmaceutical industry.

\section{WHAT IS RIGHT OR BEST, AND WHAT DOES THE FUTURE HOLD?}

It is obvious that a neurologist may be best placed to advise on diagnosis and long-term care in one patient's neurological illness, while a GP may be best placed to manage that patient's symptoms and illness in their community. The difficulty for neurology, general practice, and their patients is that, despite the continuing expansion in UK neurology, there are still not enough neurologists to meet the demand and the demand is still going up. In the context of a limited supply of specialists and an insistence on short waiting lists, the referral of too many new patients, particularly if they have nonspecific symptoms, to a neurologist potentially damages the broader care of patients with neurological illness. This is because it forces the discharge of long-term neurological patients from secondary care so that waiting list targets are met. It is unlikely that the long-term management of neurological conditions is going to become simpler or that neurological diseases will go away. Unless there is an inconceivable expansion in neurology in the UK, then neurologists continue to need GPs to gatekeep rigorously for new neurological symptoms and to manage many of the problems of the chronically ill neurological patient themselves. Otherwise, waiting list pressures mean that neurologists will be discouraged from following up patients and GPs forced to manage the complexities of modern Parkinson's disease, epilepsy, and MS medication themselves. It should be clear that both neurologists and GPs need to see and follow up patients to continue to learn from them. 
Expansion of neurology has thus far been driven by the demand for new patient appointments. If a government intends to satiate that demand then the number of neurologists needs to approach that in the US: that would mean 25 in Gloucestershire instead of four. Such a demand for new neurologists, if unchecked, could outstrip the ability of the relatively small UK-based neurology establishment to train their successors adequately. Rather worryingly, the increase so far does not appear, as yet, to have meant demonstrably better care for patients with neurological illness. ${ }^{19}$

The imposition of referral criteria is unlikely to be the answer. Neurology is full of grey areas, and the process of history taking and examination in neurology is not so precise as to categorise patients easily. GPs vary in their ability to elicit symptoms and signs and to manage neurology, and it doesn't help to judge, from the perspective of secondary care, what is or is not an inappropriate referral. On the other hand, some form of monitoring and feedback must become commonplace if expansion of neurology is limited. An audit in Brighton showed that neurologists and GPs generally agree on what is and is not the best use of neurology time and which referrals are most and least appropriate. ${ }^{10}$ Feeding back individual GPs' referral numbers relative to their peers was seen as helpful, although difficult to interpret.

The best hope for a rational system of care for neurology patients in the future may be to maintain or restore a healthy working and communicative relationship between primary and secondary care. If GPs are expected to manage neurological symptoms and disease confidently and competently, then neurologists will need to take a continuing educational and supportive role. If regional disparities in access are to be corrected, then either the existing neurologists will need to get out more, or new neurology posts should only be created at the spoke (the District General Hospitals), rather than the hub (the regional neuroscience centres), whatever the waiting list pressure at the hub. If the number of patients with a neurological condition under the care of one GP is too small to give that GP experience, then perhaps practices could rationalise care so that one interested GP could see the chronic neurology patients, look at the new patient referrals made to neurology, and periodically meet the neurologist to review both. If neurology takes too long for a 10minute consultation, then maybe two appointments could be allocated to a difficult neurology case. If GPs are going to resist NICE guidance and patient pressure to refer, then they do also need to unceasingly point out the importance of primary care in the management of patients with neurological disease.
Certainly neurology teaching in medical school could be improved, with management of the prosaic put above the esoteric. That may happen with the increasing number of neurologists. Perhaps experience in neurology should be a key constituent of GP training. GPs could spend some time in the neurology clinic (it has happened twice in the author's 10 years as a consultant), and neurologists could run clinics in GP premises. ${ }^{20}$ Neurologists might also be helped to manage MUS patients (and avoid multiple follow-up appointments) by sitting in a GP's surgery for the morning and reading Balint. ${ }^{21}$ If GPs are asked to cap referrals then it makes sense for them instead to have direct access to some neurological investigations (for example, CT, electroencephalography [EEG], neurophysiology), but with the proviso that they know the pros and cons and predictive value of each test (an EEG is not the epilepsy test, an MRI doesn't always show stroke), and what to do with the result. The provision of rapid access clinics may help, but equally could have the same effect as increasing the number of neurologists; the clinics fill and become another waiting list target that is soon exceeded.

PCTs and hospital managers have suggested 'improving the new to follow-up ratio' in neurology. This initiative is both ludicrous (because the figures above show that it is counterproductive) and disrespectful of patients with chronic disease and their doctors. Asking secondary care to 'compete' for business in the free market also makes no medical sense. When a secondary care provider benefits from increased referrals, it may be easier to encourage more referrals from the same population (particularly in a specialty that has been relatively undersupplied in the past) than it is to establish territorial advantage over other providers in the border zones. Separating purchasers from providers creates a schism between two components of a team that needs to have a single medical aim: to improve neurological health.

\section{CONCLUSION}

Whatever the relationship between neurology and primary care in recent years, the continuing waiting list pressures (and initiatives), and the huge regional disparity in access, implies that if there has been any plan it has not worked. Application of the free market has resulted in massive discrepancies in the availability of neurological care and no proof as yet that more neurology in any one area has led to better care for patients with neurological illness.

It may be futile for the Canutes among us to complain. More referral means more money for secondary care, and neurology empires can build. But while it is flattering to be in a specialty in demand, it can be equally frustrating to be unable to 
fit a patient with long-term disease into the clinic because of the need to leave space for someone who has fainted. For those who believe in the value of primary care, it is terribly disappointing to hear patients on the MS helpline saying 'My GP said to ring you because he/she doesn't know anything about MS'. As a taxpayer too I wonder if the gradual transfer of neurological diagnosis and continuing care from primary to secondary care is really a sensible way forward.

There are some positive initiatives to help primary care in the management of neurology. The appointment of experienced specialist nurses who spend time in both primary and secondary settings is helpful, although it must also be acknowledged that medical and nursing roles are different and that one cannot be expected to be a cheap replacement for the other. Some neurology departments maintain web pages with advice for managing common problems without referral, and as a way to communicate neurological developments. DeNDRoN (the Dementias and Neurodegenerative Diseases Research Network) has a clinical study group supporting neurodegenerative research in primary care, and there is an independently funded organisation (Primary Care Neurology Society) that aims to 'generate greater awareness and interest in neurology among professionals with an interest, or working, in primary care'. ${ }^{22}$ A recent publication in this journal of red, orange, and yellow flags for imaging in headache was produced by a joint primary and secondary care group; ${ }^{23}$ it would be good to see similar initiatives for funny turns, tingly legs, tremor, and memory impairment.

The overwhelming message here is that it is time that healthcare commissioners looked closely at the future of neurology in primary and secondary care; they need to tackle the geographical disparity in service provision and think beyond the free market. Does neurology go on expanding to meet the everrising demand for new referrals, or should there be active promotion of alternatives to referral? If neurology is not able to expand will the neurologist's role be forcibly limited to diagnosis and discharge? Can the GP once more be encouraged and empowered to exercise judgement (and be allowed to make the occasional mistake), or do we instead move to a position where primary care is advised to do little more for neurological patients than write referral letters?

\section{Competing interests}

The author has stated that there are none

\section{Acknowledgements}

I would like to acknowledge the very important contribution (though not necessarily the agreement) of Drs S Drewett, G Fuller, S Lipscombe, A Mancey-Barratt, A Nisbet, and D Stevens to arguments expressed here. Drs Lipscombe,
Mancey-Barratt and Nisbet also contributed to the Brighton audits.

\section{Discuss this article}

Contribute and read comments about this article on the Discussion Forum: http://www.rcgp.org.uk/bjgp-discuss

\section{REFERENCES}

1. Anonymous. Life with Parkinson's today — room for improvement. Parkinson's News - A Quarterly Bulletin for Health and Social Care Professionals 2008; 31: 9.

2. MacDonald BK, Cockerell OC, Sander JWAS, Shorvon SD. The incidence and lifetime prevalence of neurological disorders in a prospective community-based study in the UK. Brain 2000; 123(4): 665-676.

3. Rogers G. This house believes that only general practitioners with a specialist interest in epilepsy should be treating the condition. Pract Neurol 2008; 8(3): 138-140.

4. Hatcher S, Arroll B. Assessment and management of medically unexplained symptoms. BMJ 2008; 336(7653): 1124-1128.

5. Association of British Neurologists. Neurology in the United Kingdom: numbers of clinical neurologists and trainees. London: Association of British Neurologists, 1996.

6. Stevens DL. Neurology in Gloucestershire: the clinical workload of an English neurologist. J Neurol Neurosurg Psychiatry 1989; 52(4): 439-446.

7. Federation of the Royal Colleges of Physicians of the United Kingdom Census of consultant physicians in the UK, 2006. London: Royal College of Physicians, 2006.

8. Morrish P. What is happening to English neurology? Clin Med 2008; 8(6): $576-578$.

9. Latinovic R, Gulliford M, Ridsdale L. Headache and migraine in primary care; consultation, prescription and referral rates in a large population. J Neurol Neurosurg Psychiatry 2006; 77(3): 385-387.

10. Morrish PK. How do we manage referral number? J Neurol Neurosurg Psychiatry 2006; 76(12): 1396.

11. Carson AJ, Ringbauer B, Stone J, et al. Do medically unexplained symptoms matter? A prospective cohort study of 300 new referrals to neurology outpatient clinics. J Neurol Neurosurg Psychiatry 2000; 68(2): 207-210.

12. Schon F, Hart P, Fernandez C. Is clinical neurology really so difficult? J Neurol Neurosurg Psychiatry 2002; 72(5): 557-559.

13. Ridsdale L, Massey R, Clark L. Preventing neurophobia in medical students, and so future doctors. Pract Neurol 2007; 7(2): 116-123.

14. The National Collaborating Centre for Chronic Conditions. Parkinson's Disease. National clinical guideline for diagnosis and management in primary care. London: Royal College of Physicians, 2006. www.nice.org.uk/nicemedia/pdf/cg035fullguideline.pdf (accessed 23 Jun 2009).

15. The National Collaborating Centre for Chronic Conditions. Multiple Sclerosis. National clinical guideline for diagnosis and management in primary and secondary care. London: Royal College of Physicians, 2004

16. Stokes T, Shaw EJ, Juarez-Garcia A, et al. Clinical guidelines and evidence review for the epilepsies: diagnosis and management in adults and children in primary and secondary care. London: Royal College of General Practitioners, 2004

17. Royal College of Physicians, Clinical Standards Department. Long-term neurological conditions: management at the interface between neurology rehabilitation and palliative care. National Guidelines (10). London: Royal College of Physicians, 2008.

18. Caslake R, Moore JN, Gordon JC, et al. Changes in diagnosis with follow-up in an incident cohort of patients with parkinsonism. $J$ Neurol Neurosurg Psychiatry 2008; 79(11): 1202-1207.

19. Royal College of Physicians, Clinical Effectiveness and Evaluation Unit. National audit of services for people with multiple sclerosis. London: Royal College of Physicians, 2008.

20. Warlow C, Allen C, Venables G. Bringing neurology to the people. Pract Neurol 2008; 8(4): 208-210.

21. Balint M. The doctor, the patient and his illness. 2nd edn. London: Churchill Livingstone, 2008.

22. Primary Care Neurology Society. The society. http://www.pcns.org.uk/thesociety.asp (accessed 23 June 2009).

23. Kernick DP, Ahmed F, Bahra A, et al. Imaging patients with suspected brain tumour: guidance for primary care. Br J Gen Pract 2008; 58(557): $880-885$. 